\title{
The Radio Sonde*
}

\section{W. H. PICKERING $\dagger$, ASSOCIATE, I.R.E.}

Summary-The radio sonde has been developed in recent years as a practical instrument for transmitting information from the stratosphere. This paper discusses some of the problems connected with the application of the radio-sonde principle to the radio meteorograph and also to the cosmic-ray radio sonde.

\section{INTRODUCTION}

W ITHIN recent years techniques have been successfully developed for the automatic transmission of meteorological and other information from instruments sent into the stratosphere by free balloons. This article is intended to review some of the problems encountered with such instruments, and, in particular, to describe the successful application of the radio sonde for cosmic-ray observations.

About six years ago latex-rubber balloons were first made commercially available in large sizes. This meant that relatively large weights could be lifted to great altitudes at a reasonable expense, and so greatly extended the possible field of usefulness for radio exploration of the stratosphere. These latex balloons are produced in sizes up to a weight of rubber in the balloon of 700 grams. Such a 700-gram balloon may be inflated with hydrogen to lift about 1500 grams at a rate of almost 1000 feet per minute to an altitude of 70,000 feet or more. If the balloon is inflated to a larger initial lift, then the bursting altitude will be correspondingly reduced. If the balloon is inflated to give a very slow ascent there will not be much gain in the maximum altitude reached. This is probably because one of the limiting factors in the bursting of the balloon is the fact that the thin, stretched rubber deteriorates rapidly under the action of sunlight and ozone.

When a flight is being made to the maximum altitude, the ascent will require about 90 minutes. During this time the balloon may drift a considerable distance. In temperate latitudes the drift will usually be to the east and may amount to 75 miles. At lower latitudes conditions may be found where light and variable winds are encountered and the drift may be only 10 or 20 miles. However, the probability that no wind will be encountered at any altitude is very small, although in the author's experience, flights made in Southern Mexico in December, 1941, consistently showed no wind between a slight surface wind only a few hundred feet thick and a westerly wind at about 30,000 feet.

The descent after the maximum of the flight has been reached may be accomplished either by using a parachute or a second balloon. When two balloons with approximately equal lift are used for a flight, the probability that both balloons will burst simultaneously is

\footnotetext{
* Decimal classification: R539. Original manuscript received by the Institute, February 1, 1943.

† California Institute of Technology, Pasadena, California.
}

very small, and thus the remaining balloon will lower the instrument safely to the ground. In this case there is an advantage as far as recovery of the instrument is concerned, in that the grounded instrument with the balloon attached is a fairly conspicuous object.

Since the descent may take almost as long as the ascent, the equipment should be designed to operate for 3 to 4 hours, and to transmit a readable signal at the end of this time to a range of 150 miles.

\section{Design of the Transmitter}

The factors affecting the design of the transmitter may be listed as follows:

(a) Weight. Obviously the weight must be as small as possible, particularly as the transmitter is merely a means for passing on the information obtained by the instrument.

(b) Power supply. The power required, both filament and plate power, must be kept to a minimum.

(c) Operation at low temperature. The frequency shift at temperatures as low as -60 degrees centigrade should be small.

(d) Operation at low pressure. No voltages should be high enough to produce corona difficulties at air pressures of a few millimeters.

The choice of wavelength is. not restricted by the transmitting antenna for this may readily be made of almost any length. The weight of the antenna wire is certainly small, and it can act as a part of the connection to the balloon. The wavelengths used in soundingballoon flights are chosen with regard to such other factors as, for example, the wavelengths assigned for such use, the physical size and weight of the resonant circuits in the transmitter, and the wavelength at which the transmitting tubes will operate most efficiently. If the receiving station is to be reasonably portable, considerations of receiving antenna design may also govern the choice of wavelength.

The transmitting antenna is nearly always a vertical half-wave antenna. Such an antenna is simple from a mechanical standpoint and it radiates equally well in all horizontal directions. There is no radiation vertically downward, but, as mentioned above, the chance of the balloon remaining vertically over the receiving station is very small. The radiation is a maximum in the horizontal direction, which is desirable because, when the balloon is at a great distance, the angle will of necessity be small.

A simple calculation shows that if vertical dipole antennas are used at both transmitter and receiver, and if the effect of the ground is neglected, the received signal strength is given by $E=(k / h) \cos ^{2} \theta \sin \theta$ where $\theta$ is the elevation angle to the balloon and $h$ is the height of the balloon over a plane earth. 
For a constant $h$ the maximum received signal will occur when $\theta=35$ degrees. See Fig. 1.

If steady signals are to be received at the ground station there must be no fluctuation in radiated signal as the balloon and instrument rotate about a vertical axis or swing back and forth about a horizontal axis. The effect of rotation will be eliminated by insuring that the vertical antenna is the only radiator. This is difficult to attain at ultra-high frequencies without complete shielding of the transmitter. Swinging may give rise to two effects. First, the mechanical motion

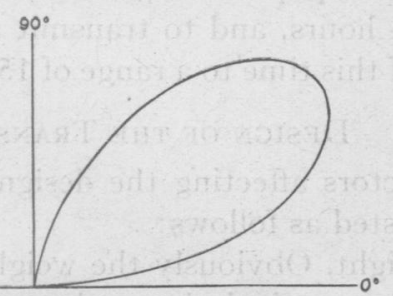

Fig. 1-Polar diagram showing the received signal at a ground station from a transmitter on a balloon at a constant height over a plane earth, as a function of the elevation angle of the balloon. Vertical dipole antennas at both transmitter and receiver and ground reflections neglected.

may cause a shift in transmitter frequency, and second there will be a change in the polarization and signal radiated in the direction of the receiver, with a corresponding change in receiver output. This can only be eliminated by eliminating the swinging as much as possible. A long supporting string between balloon and instrument will help a great deal, and the use of the antenna as a part of the supporting system will make a further improvement.

The fractional change in received signal due to a swing through an angle $\delta$ on each side of the vertical may be shown to be approximately $2 \delta \tan \theta$, where $\theta$ is the elevation angle. Hence the effect of swinging is more pronounced at large elevation angles.

A typical radio-sonde transmitter is illustrated in Fig. 2. This transmitter operates on 180 megacycles (1.67 meters) and therefore acorn-type tubes are used. The 958 is chosen because it is more conservative of filament power, and will give about the same output as the 955 with a given plate voltage. Since the transmitter is expected to operate for only a few hours, and since it may deliver power for only a fraction of this time, the oscillator tube may be operated safely at a considerable overload. The circuit of Fig. 2 is a standard Colpitts' oscillator circuit. Trimmer condensers are used for tuning adjustments. The half-wave antenna is attached through a short flexible lead directly to the tank coil. In a cosmic-ray radio sonde, where the transmitter is keyed with short pulses, this oscillator has been used with 1.7 volts on the filament and 135 volts on the plate. ${ }^{1}$

Radio-sonde transmitters operating in the ultrahigh-frequency region may be expected to have much

${ }^{1}$ H. V. Neher and W. H. Pickering, "A cosmic-ray radio sonde," Rev. Sci. Instr., vol. 13, pp. 143-147, April, 1942. the same design as shown in Fig. 2. At wavelengths of a few meters, tubes such as the 30 , or the $1 \mathrm{C} 5$, or the newer small tubes such as the 1S4 are satisfactory.

The power supply for the transmitter frequently will contribute the major part of the weight of the complete radio sonde. Batteries offer the only practical means of supplying this power. Both primary and secondary batteries are used. Much can be said for and against each of these.

The primary battery is always ready for use, there is no danger from spilled acid, it can be mounted wherever convenient in the instrument, and can even be left connected with a switch to be closed for the actual flight or for testing. It has the disadvantage of short shelf life, particularly in the small sizes of B battery. Small dry batteries have been constructed commercially for radio-sonde use. The smallest sizes of batteries used in camera-type radio receivers are also useful for radio-sonde applications.

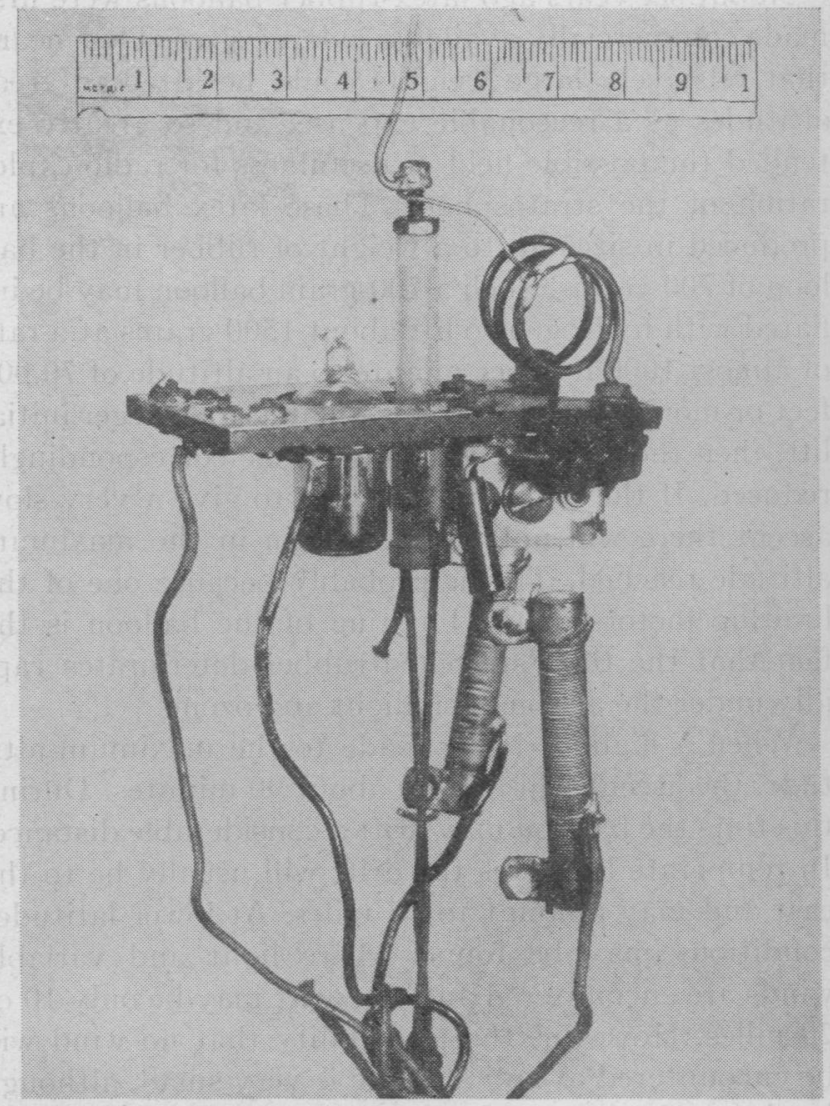

Fig. 2-A radio-sonde transmitter for a frequency of 180 megacycles. Signals from this transmitter have been received at distances up to 200 miles.

Storage batteries have advantages of indefinite shelf life when stored dry, constancy of voltage during discharge, operation at very low temperatures, and light weight. By using small sections of the very thin plates used in airplane-type storage batteries, a battery can be assembled which has less than half the weight of the equivalent dry-cell battery. The disadvantages of storage batteries are primarily the mechanical problems of 
construction of the B battery. The cells must be designed so as to avoid internal short circuits or open circuits, and also they should be almost completely enclosed to prevent acid from spilling or short-circuiting the battery.

In some early work with radio-equipped balloons, power was supplied from an A battery alone, with the plate power provided by a vibrator and transformer. ${ }^{2}$ The modern developments in lightweight $\mathrm{B}$ batteries, however, have resulted in their use in all recent work. Although it might be possible to make a vibrator and transformer unit of comparable weight with the B battery, the additional complication in the circuit would be hard to justify. On the other hand some cosmic-ray equipment requires a voltage of 1000 to 1500 volts with a negligible current drain, for a Geiger counter voltage supply. Here the vibrator and transformer offer a very satisfactory solution. A high-voltage supply of this sort has been previously described. ${ }^{3}$ It uses a transformer, rectifier tube, and filter circuit sealed in an airtight can to avoid corona difficulties and it is operated by a 4 -volt storage battery and a buzzer. The current drain is about 50 milliamperes at 4 volts, plus the rectifier filament which requires about 40 milliamperes at 1 volt. The total weight, including battery power for three hours operation, is 650 grams. During the operating period the high-voltage output will change about 50 volts out of 1200 .

\section{The Meteorograph}

The application of the radio-sounding balloon to meteorological problems requires that pressure, temperature, and humidity measurements be made as frequently as possible during the balloon flight. An ideal meteorograph would complete the cycle of readings every 30 seconds and would have an accuracy of 3 millibars in the pressure, 0.5 degree centigrade in temperature, and 5 to 10 per cent in the relative humidity. Two basic principles have been used for converting the readings of the meteorological elements into modulation of the transmitter. These are:

(1) The use of the Olland telemeteorograph principle.

(2) Modulation directly by the variations, either mechanical or electrical, of the meteorological elements, together with some switching mechanism for connecting the elements in sequence to the transmitter.

The Olland principle is illustrated schematically in Fig. 3. Expansion of the aneroid barometer causes the arm $A$ to move in the region between points $B$ and $C$. A contact $\operatorname{arm} D$ is turned uniformly by a clockwork or motor mechanism and makes contact in turn with $B, A$, and $C$. At each contact a circuit is closed which then operates a relay or, in this case, completes the

${ }^{2}$ W. R. Blair and H. M. Lewis, "Radio tracking of meteorological balloons," Proc. I.R.E., vol. 19, pp. 1531-1561; September, 1931.

${ }^{3}$ H. V. Neher and W. H. Pickering, "A lightweight high voltage supply for Geiger counters," Rev. Sci. Instr., vol. 12, pp. 140-142; March, 1941. plate circuit of the transmitter. Thus a signal is radiated for the duration of the contact. At the ground station these signals are recorded on a uniformly moving paper tape. The distance on the tape between the signals corresponding to contacts $B$ and $A$ will then be a measure of the barometric pressure. Contact $C$ will serve as a check on the uniformity of clock speed, or an additional point for measurement in the event contact $B$ is not received properly. The temperature and humidity measurements are made similarly by inserting additional contact arms into the cycle and using the mechanical motion of a bimetallic strip thermometer and a hair hygrometer to drive them. With reasonable care in the design such a unit can be constructed to give a sequence of signals which is readily identified, and which will have good accuracy. The final limitation on accuracy, if the unit is driven by a clock, is the fact that the clock arm moves forward in jumps with the

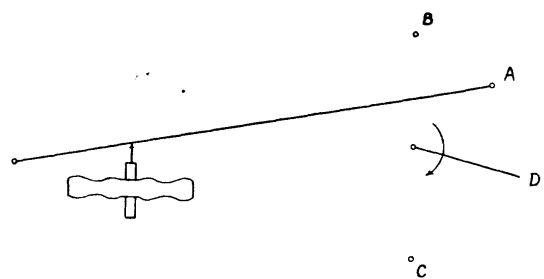

Fig. 3-The Olland telemeteorograph principle.

beats of the balance wheel. For a clock beating 5 times a second, and a clock arm rotating once in 30 seconds, the arm actually moves forward $1 / 150$ of a revolution at each jump. Hence, if conditions were as sketched in Fig. 3, where the total movement of $A$ is less than half a revolution of the $\operatorname{arm} D$, the accuracy in the reading of $A$ would correspond to about $1 / 75$ of an atmosphere change in pressure or about 15 millibars. This is not good enough. To improve the accuracy, the clock can be made to beat faster, ${ }^{4}$ or the design may be modified to allow the travel of the contact arms to correspond to several revolutions of the clock..$^{5,6}$

Several different types of radio meteorograph have used the second method of modulating the transmitter. For example, in the Diamond-Hinmann meteorograph, used by the United States Weather Bureau, the temperature and humidity elements are designed to produce changes of electrical resistance with changes of temperature and humidity. ${ }^{7}$ The transmitter is modulated at an audio frequency which is made a function of the circuit resistance, and thus switching the temperature unit in to the circuit will produce an audiofrequency modulation whose frequency will be a

${ }^{4}$ O. C. Maier and L. E. Wood, "The Galcit radio meteorograph," Jour. Aero. Sci., vol. 4, pp. 417-422; August, 1937.

${ }^{5}$ K. O. Lange, "The 1935 radio-meteorographs of Blue Hill Observatory," Bull. Amer. Met. Soc., vol. 17, pp. 139-140; May, 1936.

${ }^{6} \mathrm{~K}$. O. Lange, "The 1936 radio-meteorographs of Blue Hill Observatory," Bull. Amer. Met. Soc., vol. 18, pp. 107-126; March, 1937

7 C. B. Pear, "Radio sounding in the United States," Electronics, vol. 16, pp. 82-85; January, 1943. 
measure of the temperature, and similarly, when the humidity unit is in the circuit the audio frequency received is a function of the relative humidity. The switching is done by the barometer element. As the pressure changes, the arm connected to the aneroid barometer moves over a contact strip and switches the other elements alternately into the modulation circuit. The pressures at which the switching takes place are determined in a calibration. At definite pressure intervals a fixed resistor is switched into the circuit to check on the audio frequency transmitted and to assist in the identification of the switching pressures. The basic circuit of the oscillator and modulator is shown ${ }^{8,9}$ in Fig. 4.

Tube $T_{1}$ oscillates at a relatively low radio frequency. Because of the long time constant $R C$ in its grid circuit, the oscillations are periodically blocked

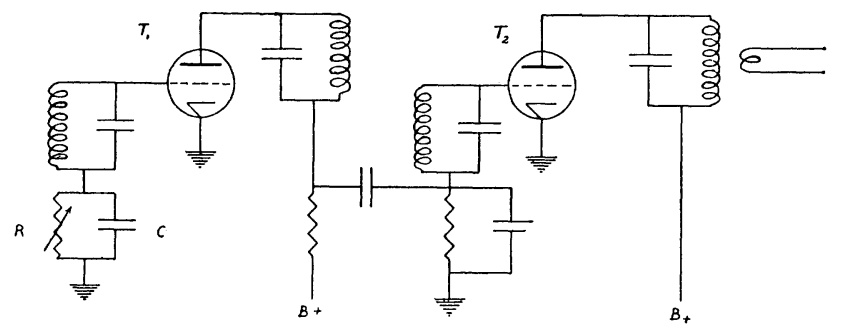

Fig. 4-The basic circuit of the Diamond-Hinmann radio-meteorograph transmitter.

at an audio frequency determined by $R C$. This blocking produces periodic impulses at the grid of $T_{2}$ and hence modulates the radio-frequency output of $T_{2}$.

One of the earliest successful radio meteorographs was due to Moltchanoff. ${ }^{10} \mathrm{He}$ used an arrangement in which the mechanical movement of the meteorological elements was converted into a coded sequence of transmitted dots and dashes. The switching and keying were provided by a wind-driven fan.

Meteorographs have been developed in which the change in the meteorological elements is converted into a change in the transmitted radio frequency. In the Väisälä instrument used in Finland ${ }^{11}$ the meteorological elements are mechanically connected to variable condensers whose capacitances are thus a function of the quantity to be measured. A fan-driven switch connects each condenser in turn to the transmitter tank circuit and thus the radio frequency transmitted is made to measure the desired quantities. An additional fixed condenser is also switched into the circuit periodically in order to serve as a reference frequency.

${ }^{8}$ H. Diamond, W. S. Hinmann, F. W. Dunmore, and E. G. Lapham, "Ān improved radio sonde and its performance," Nat. Bur. Stand. Jour. Res., vol. 25, pp. 327-367; September, 1940.

${ }^{9} \mathrm{H}$. Diamond, W. S. Hinmann, F. W. Dunmore, "A method for the investigation of upper-air phenomena, and its application to radio meteorography," PROC. I.R.E., vol. 26, pp. 1235-1265; October, 1938.

${ }^{10} \mathrm{P}$. Moltchanoff, "Drei Jahre Aufstiege von Radiosonden im Institute der Aerologie, Sloutzk, U.S.S.R.," Meteor. Zeit., vol. 50, p. 428; November, 1933.

11 V. Väisälä, "Eine neue Radiosonde," Mitt. d. Met. Inst. d. Univ. Helsingfors, vol. 29, pp. 1012-1029; 1935.
The meteorograph of Duckert, Germany, uses a variable condenser driven by a bimetallic strip and permanently connected to the transmitter. ${ }^{12,13} \mathrm{By}$ careful design, this is made to be the chief factor affecting transmitter frequency, and thus a continuous record of temperature is obtained. The pressure element moves an arm over a contact strip and interrupts the transmitted signal at certain definite pressures. Humidity is not recorded.

\section{Receiving Equipment}

Receivers used for radio-sonde work normally will be designed along conventional lines except for the recording mechanism necessary. At the higher frequencies superregenerative receivers usually have been used because of their sensitivity, broad tuning, freedom from pulse interference, and inherent automatic-volume-control action.

The recording technique for a radio meteorograph should be such that measurements of the meteorological elements can be made during the flight, and the complete flight record completed within an hour of the end of the flight. Relatively unskilled personnel, from a radio point of view, should be able to handle all ordinary adjustments on the recorder. The accuracy of the recorder should be at least as good as that of the transmitter.

When the Olland principle is used the recording technique is very simple. The output from the receiver is amplified sufficiently to operate a relay which then makes a mark on a moving strip of paper. The accuracy of measurement depends on the precision with which the speeds of the clock at the transmitter, and the motor driving the recorder, can be held constant. Because of the power necessary to make the various contacts and because of the wide variation in temperature to which it is necessarily exposed, it is difficult to keep the transmitter clock running uniformly. Experiments have been made with small electric motors, gravity drives, and wind drives, as substitutes for a mechanical clock, but these have not proved very successful. In spite of this problem, the simplicity and directness of the Olland principle at both the transmitting and the recording ends, makes a very satisfactory system for radio-sonde measurements.

The Diamond-Hinmann system requires a recording audio-frequency meter at the receiving station. The discontinuities in the frequency record are then corelated with pressures. The precision of the temperature and humidity measurements is determined by the accuracy with which the variation of resistance introduced by the temperature and humidity elements is known and reproducible, and the accuracy with which

12 P. Duckert, "Das Radiosondenmodell Telefunken und seine Anwendung," Beitr. z. Phys. d. fr. Atmosph., vol. 20, pp. 303-311; 1933.

${ }^{13}$ K. O. Lange, "Other radio meteorographs," Bull Amer. Met. Soc., vol. 16, pp. 233-236; 267-271; 297-300; October, November, December 1935 . 
the audio modulating frequency is determined by this resistance. The precision of the measurement of this audio frequency is also a limiting factor. An accuracy of 0.5 degree centigrade in a total range of about 100 degrees centigrade is 0.5 per cent, and this is almost certainly approaching the limit of any recording electrical meter. The pressure determination depends, in the last analysis, on the accuracy and reproducibility of the calibration. The movement of the barometer arm across the contact strip must be free of any irregularities.

The meteorographs depending on a variation of the transmitted radio frequency require a wide frequency channel for successful operation. Assuming that a frequency deviation is to be measured rapidly and accurately to 0.5 per cent, it is obviously desirable that 0.5 per cent of the maximum deviation be large compared with the deviations of the carrier frequency produced by tube variations, change of battery voltage, change of temperature, etc. These deviations may well amount to 0.1 per cent of the carrier frequency in themselves, and thus it appears that the frequency deviation produced by the variation of the meteorological elements should approach the magnitude of the carrier frequency. In the Väisălä meteorograph, a comparison with a reference condenser is made periodically. This makes the deviation requirement much less stringent, but still requires a wide channel for accurate measurement. The recording technique used with these instruments consists essentially in keeping a receiver tuned to the signal and periodically noting the tuning.

It is not the intent of this paper to discuss the merits of the various instruments from a meteorological point of view, or to discuss the mechanical design of a successful radio meteorograph. These are problems for the meteorologist and the instrument maker. However, one feature of the problem does concern the radio engineer. A practical instrument must be used under all sorts of field conditions. It must be released under bad weather conditions, it may fly through snow and rain, ice may form on the instrument. To release a balloon in a high wind is well nigh impossible unless a very short connection between instrument and balloon is used. From the radio point of view this is unfortunate because the instrument will swing violently during a considerable portion of the flight. Thus, in designing a radio-sonde transmitter, although weight must be counted in grams, at the same time, the unit must have sufficient mechanical stability to withstand considerable abuse.

\section{A Cosmic-Ray Radio Sonde}

The radio-sonde technique has been used for cosmicray observations at great altitudes. Briefly the electrical problems may be stated thus. The cosmic-ray impulses from one or more Geiger counters, are to be amplified until they can modulate the transmitter. The number of cosmic-ray impulses may be of the order of 1000 per minute with a statistically random-time distri- bution, and thus their number must be scaled down electrically at either the transmitter or the ground station before they can be recorded. If, as seems logical, this scaling is done on the ground, the length of the transmitted pulse must be of the order of 100 microseconds. A barometer signal must also be sent periodically.

An instrument to satisfy these requirements will be much heavier and more complicated than a radio meteorograph. The instrument used by the author, Fig. 5, weighs about 3500 grams complete with protective coverings and lines as compared with weights of less than 1000 grams for a radio meteorograph. The component parts of this instrument are as follows: The Geiger counters to detect the cosmic rays require a power supply delivering about 1200 volts direct current. Each pair of counters is connected to a three-stage resistance-coupled amplifier. The outputs from the third stages are then paralleled in such a manner that only coincident impulses in the two amplifiers are passed. These impulses are further amplified with a power-amplifier tube and modulate the transmitter. The final modulation and pulse-shaping circuit is shown in Fig. 6. The barometer signals are derived from an Olland cycle, and are fed into the circuit as indicated in Fig. 6. The transmitting unit requires a total tube complement of 9 tubes.

The received signal consists of an irregular succession of very short pulses, interrupted by audio signals at about 5000 cycles lasting for about 1 second. The superregenerative receiver unfortunately cannot be used because it fails to respond to very short pulses. This is shown by its ability to suppress automobile ignition noise, whereas the desired signal actually has very much the same appearance as ignition noise. With such a short signal, a superheterodyne receiver must be used. Furthermore, a receiving location that is free from ignition interference is absolutely necessary. The desired information is the number of cosmic-ray impulses per minute as a function of barometric pressure, hence the impulses must be counted and recorded. To do this an electrical scaling circuit is needed so that every $n$th pulse may be fed into a recording relay and written on a paper tape. The basic scaling circuit is the vacuum tube "scale-of-two" circuit. ${ }^{14}$ Six stages in cascade give a total scaling factor of 64 which may be adjusted in powers of two by a selector switch. Figs. 7,8 , and 9 illustrate, respectively, the circuits of the input pulse equalizing circuit, the scale of two, and the output pulsing circuit which operates the recording pen. This pen writes on a paper tape moving at about 15 centimeters per minute. Near the beginning of a flight the scaling circuit is switched to the "multiplyby-1" position, and each incoming pulse is recorded as a transverse line on the tape. These pulses are statistically random in time and thus appear quite irregular. When the audio frequency from one of the Olland

${ }^{14} \mathrm{H}$. Lifschutz and J. L. Lawson, "A triode vacuum tube scaleof-two circuit," Rev. Sci. Instr., vol.9, pp. 83-89; March, 1938. 


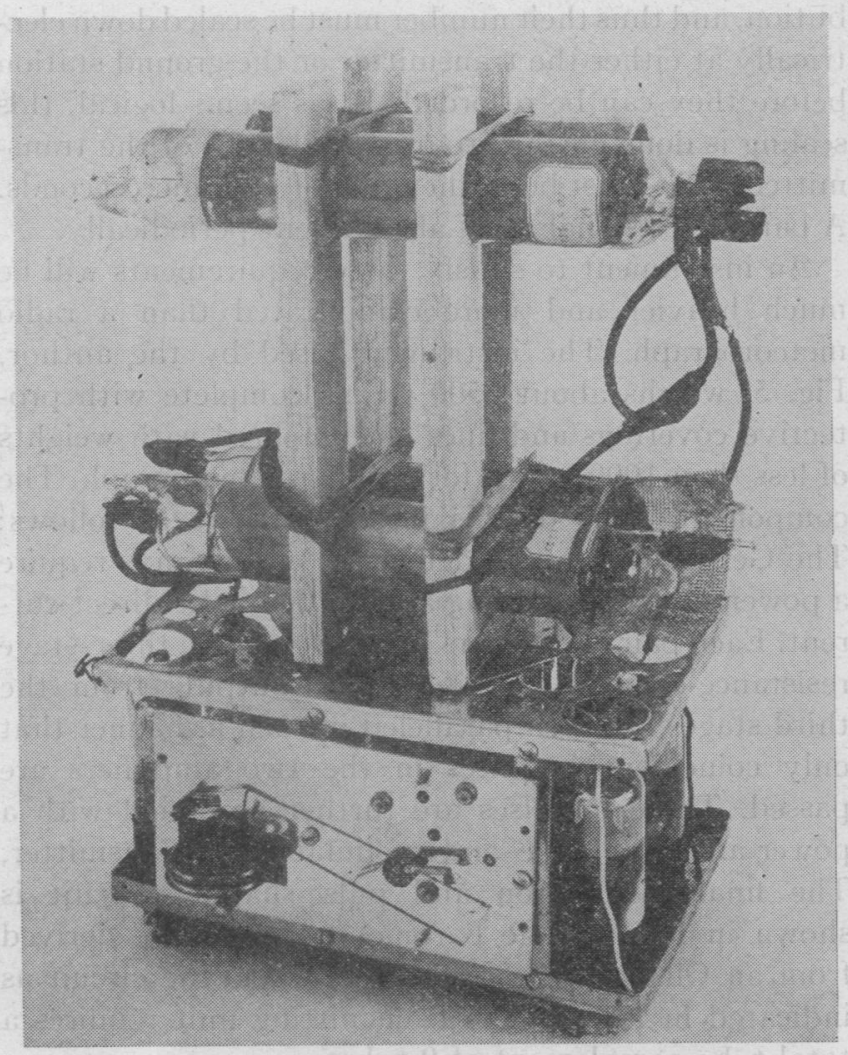

Fig. 5-A cosmic-ray radio sonde. The transmitter of Fig. 2 is used with this instrument. The four Geiger counters are held in a balsawood frame above the aluminum chassis. In the center of the unit is a 1-pint paint can containing the high-voltage supply for the counters. The barometer and thermometer unit is covered with an aluminum shield during the flight. Batteries are contained in a box which plugs into a socket beneath the chassis. This particular instrument was released at Pocatello, Idaho, and recovered in Wyoming about 100 miles to the east.

cycle contacts is received, the recording pen is deflected and remains deflected for the duration of the signal, because it is unable to follow the rapid succession of signals. Thus the barometer signals appear as a deflection of the pen for about 1 second or 3 millimeters on the tape. These marks are readily distinguished from the sharp cosmic-ray signals. As the instrument gains in altitude and the signals become more rapid, one or more scaling stages are switched into the circuit. When scaling factors of 16 or higher are used, the statistical fluctuations are almost completely removed and the cosmic-ray record appears as a

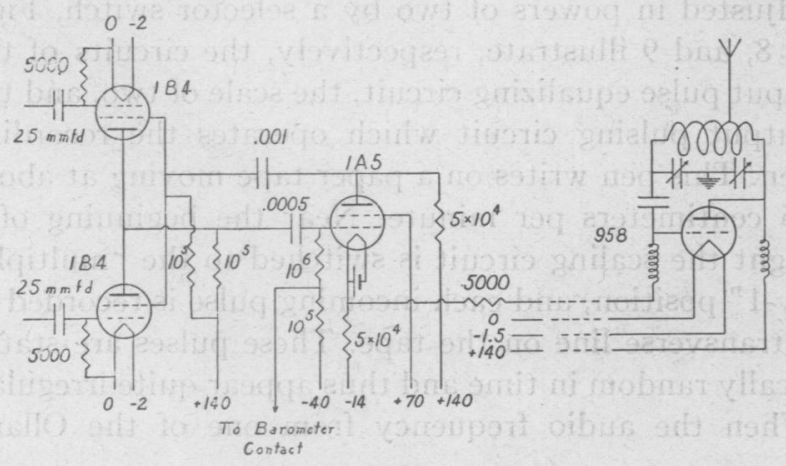

Fig. 6-The modulation circuit for the transmitter of the cosmic-ray radio sonde. succession of uniformly spaced marks on the tape. Even at high scaling factors, the recording pen still gives a steady deflection on the barometer signals.

The flight conditions in making cosmic-ray observations can be made much more favorable than in the meteorological case, for only the barometric pressure is measured and thus the instrument temperature can

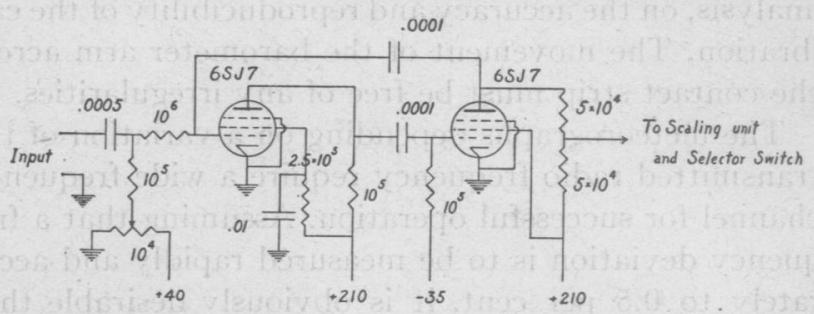

Fig. 7-Input pulse-shaping circuit for the electrical scaling circuit.

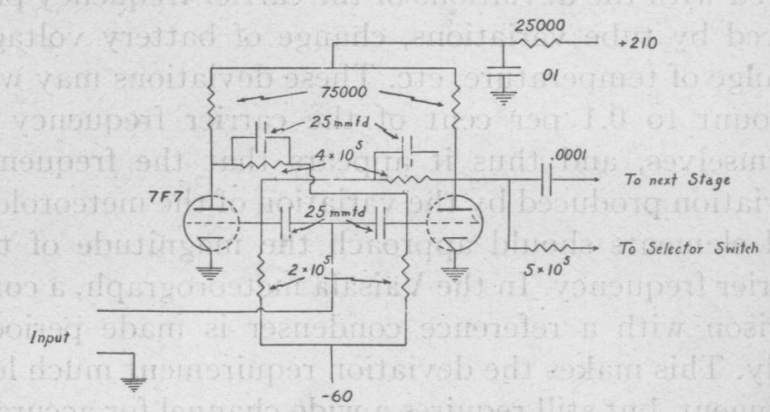

Fig. 8-One of the six "scale-of-two" circuits.

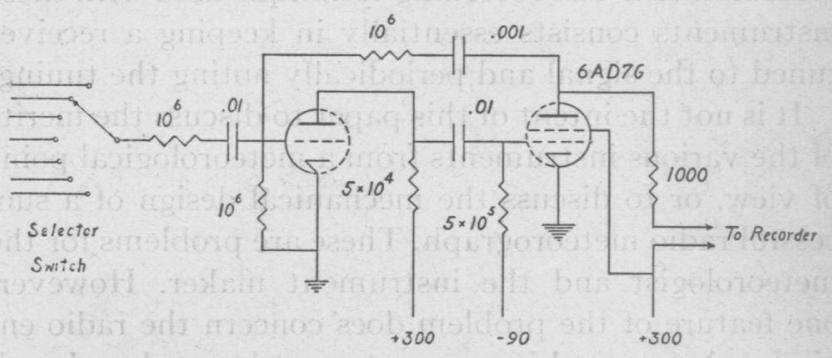

Fig. 9-The output circuit of the scale of 64 counter.

be kept close to ground temperature, and also, good weather conditions can be used for all flights. Control of instrument temperature is effected by placing the instrument in a protecting bamboo basket and then wrapping the basket tightly in cellophane. In this way the sun keeps the instrument warm and cold air is not allowed to come in contact with it. By using a wrapping that is partly clear and partly dark cellophane, the inside temperature can be controlled quite accurately. Clear cellophane alone would make the instrument much too hot. In actual flights the temperature as measured by a bimetallic strip included in the O1land cycle first-increases about 5 degrees centigrade, then drops to about 5 degrees centigrade below its initial value and finally, at very high altitudes, increases again towards its initial value.

\section{CONCLUSION}

The development of practical radio-sonde transmitters has opened up a new field for scientific exploration, 
the stratosphere. Already the meteorological observations of pressure, temperature, and humidity at altitudes far beyond the reach of any airplane, are an everyday occurrence. In the not-too-distant future it is reasonable to expect that accurate direction finding will give the meteorologists data on wind velocities at these altitudes. The cosmic-ray observations have proved to be of the greatest value in the physicist's search for knowledge concerning these most energetic of all known radiations. Measurements of almost any type can be adapted to the radio-sonde technique. For example, photoelectric measurements could be made in several different ways. The radio-sonde transmitter is essentially a telemetering system and it may be used accordingly. Indeed, lightweight, battery-operated transmitters of the radio-sonde type may prove useful for such purposes on the ground as well as in the air.

Future developments in radio-sonde techniques, besides involving the applications of the technique to specific new problems, will call for the development of more satisfactory lightweight power sources, improvement of operating efficiency for the higher frequencies, development of balloons capable of reaching higher altitudes. Improvements are always possible in any instrument and, to take the important example of the radio meteorograph, much remains to be done to make this a completely stable and reliable field instrument.

\section{Space-Current Flow in Vacuum-Tube Structures*}

\section{B. J. THOMPSON†, FELLOW, I.R.E.}

\begin{abstract}
Summary-From well-known formulas for space-current in diodes and for amplification factor in triodes, interelectrode capacitance, plate current, and potential distribution in triodes and multigrid tubes are determined through use of the concept of planes of equivalent potential. By the same means, amplification factor in multigrid tubes is derived.
\end{abstract}

\section{INTRODUCTION}

$\mathbb{V}$ ACUUM-TUBE design is a subject which has intrigued many workers, largely, one may suspect, because it has presented many possibilities for ingenious methods of analysis. The resulting knowledge of the design factors which determine the various performance characteristics of tubes is quite complete and is expressed in terms which can readily be applied to practical tube-design problems. In spite of this state of the art, the general tendency is to make use of the more "scientific" phases of tube design to aid qualitative understanding rather than to supply specific design information. In this paper, the writer presents some of the concepts of vacuum-tube analysis which he has found informative and useful.

First, space-current flow in diodes will be discussed. Then, methods will be presented for reducing triodes and multigrid tubes to equivalent diodes. Amplification factor, interelectrode capacitance (cold), and electron transit time will be covered. The writer claims little originality and no novelty in this material. Some effort has been made to give credit to the proper sources.

\section{Ideal Case}

\section{A. Diode Theory}

The simplest vacuum tube is the diode. The behavior of multielectrode tubes may be described most

* Decimal classification: R131. Original manuscript received by the Institute, March 16, 1943. With the exception of the introduction, this material was prepared for use within the RCA Manufacturing Company, Inc., duringthe fall of 1937. Similar material was presented by the author in July, 1937, at the Electronics Institute, University of Michigan, Ann Arbor, Michigan.

$\dagger$ RCA Laboratories, Princeton, New Jersey. readily in terms of the behavior of a diode. For these reasons our treatment will start with the diode.

In the ideal diode, electrons are emitted from the cathode in unlimited numbers at zero velocity and a part of these are drawn over to the anode under the influence of the positive field established by its potential.

In Fig. $1, K$ represents the infinite plane cathode at zero potential and $A$ the plane anode at a positive potential $E_{b}$ spaced a distance $d_{k p}$ from the cathode. Let us suppose first that no electrons are emitted from the cathode. The potential distribution will then be as represented by the line $a$, the gradient at all points being $E_{b} / d_{k p}$. If now the cathode begins to emit a limited supply of electrons, all of these electrons will be drawn to the anode. The electrons move at a finite velocity and, therefore, there is a certain number of them in the space at all times. The field set up by the negative "space charge" of these electrons acts to depress the potential in the space below that of the first condition, increasing the field near the anode and decreasing it near the cathode. This condition is shown by line $b$.

If the rate of emission of electrons is continually increased, all of the emitted electrons will be drawn to the anode and the gradient at the cathode continually reduced until the gradient reaches zero. Since the electrons are assumed to be emitted with zero velocity, they can not move against a retarding field; therefore, there will be no increase in anode current with further increase in emission beyond this point. The condition of zero gradient at the cathode is represented by the line $c$ in Fig. 1 .

The mathematical analysis of the ideal parallelplane case is quite simple. It will be presented here as an example of this type of analysis.

Poisson's equation in rectangular co-ordinates is

$$
\partial^{2} E / \partial x^{2}+\partial^{2} E / \partial y^{2}+\partial^{2} E / \partial z^{2}=-4 \pi \rho .
$$

Since there is no gradient in directions parallel to the cathode and anode, the equation becomes simply 